\title{
Infección diseminada por BCG en la Región de Los Lagos, Chile: Reporte de cinco casos clínicos
}

\author{
ALEXIS STRICKLER P.*, M. LINA BOZA C.**, BENITO GONZÁLEZ M.***, \\ GISELA MÁRQUEZ M.**** y JACINTA BUSTAMANTE*************
}

\section{Disseminated infection by BCG in Región de Los Lagos, Chile: Five cases report}

The bacillus Calmette-Guérin (BCG) is the attenuated strain of Mycobacterium bovis used in developing countries for preventing serious forms of tuberculosis. The neonatal BCG vaccine is applied in countries with high prevalence of tuberculosis. Most of the vaccinated individuals develop no adverse reactions; although, some subjects show side effects due to a host altered immunity. These reactions range from a simple adenomegaly in the same side of BCG vaccine inoculation, to a spread infection, often fatal. A regional or systemic spread has been described in patients with secondary or primary immunodeficiencies and partial or total genetic defects of interleukin IL-12/23 and IFN- $\gamma$ called as a whole "Mendelian susceptibility to mycobacterial infections" (MSMD). We describe five patients infected with M. bovis BCG-diagnosed between 1995-2008, at the base hospital in the city of Puerto Montt, Región de Los Lagos, Chile. These patients have the clinical course of MSMD.

Key words: Calmette-Guérin (BCG), adverse reactions, spread infection.

\section{Resumen}

El bacilo Calmette-Guérin (BCG), es la cepa atenuada de Mycobacterium bovis utilizada en países en vías de desarrollo para la prevención de formas graves de tuberculosis. La vacuna BCG neonatal se administra en países con alta prevalencia de la enfermedad. La mayoría de los vacunados no presenta reacciones adversas, algunos evidencian reacciones secundarias a una inmunidad alterada del huésped. Dichas reacciones varían desde una simple adenomegalia ipsilateral a la inoculación de BCG, hasta una infección diseminada, a menudo mortal. La infección diseminada se ha descrito en pacientes inmunodeficientes secundarios, primarios y en pacientes con defectos genéticos del eje interleuquina 12-23 (IL12/23)-interferón gama (IFN- $\gamma$ ) denominados "Síndrome de predisposición mendeliana a infecciones micobacterianas" (PMIM). Describimos cinco pacientes con infección por M. bovis-BCG diagnosticados entre 1995-2008, en el Hospital Base de Puerto Montt, Región de Los Lagos, Chile que cumplen con los criterios del PMIM.

Palabras clave: Calmette-Guérin (BCG), reacciones adversas, infección diseminada.

\footnotetext{
* Servicio de Pediatría, Hospital de Puerto Montt, 5501647 Región de los Lagos, Chile.

** Servicio de Pediatría y Unidad de Neumología Pediátrica, Hospital San Borja-Arriarán, 8360160 Santiago, Chile.

*** Unidad de Inmunología, Hospital Luis Calvo Mackenna, 7500539 Santiago, Chile.

**** Servicio de Cirugía Infantil, Hospital Puerto Montt, 5501647 Región de Los Lagos, Chile.

***** Laboratorio de Genética Humana de Enfermedades Infecciosas, Institut National de la Santé et de la Recherche Médicale, U550, 75015 Paris, Francia, UE.

****** Universidad Paris René Descartes, Facultad de Medicina Necker, 75015 Paris, Francia, UE.
} 


\section{Introducción}

La vacuna BCG neonatal es usada en los países con alta prevalencia de TBC, por su eficacia documentada en prevenir las formas letales de la enfermedad, aunque no previene la infección en otras localizaciones. Es una vacuna barata y con un perfil de seguridad aceptable. La mayoría de los individuos vacunados no presenta reacciones adversas, aunque algunos pacientes presentan reacciones secundarias, que varían desde una reacción local pequeña, o una adenomegalia localizada al mismo lado de la inoculación BCG, hasta una infección diseminada a menudo mortal.

Describimos cinco pacientes con infección por M. bovis-BCG diagnosticados entre 19952008, en el Hospital Base de Puerto Montt, Región de Los Lagos, Chile, que cumplen con los criterios del Síndrome de predisposición mendeliana a infecciones micobacterianas (PMIM) $)^{1-5}$ (Figura 1).

Estos pacientes son de origen blanco hispano, residentes de esta área, sin consanguinidad conocida ni antecedentes familiares de inmunodeficiencia o muertes de causa no precisada con inmunizaciones completas para la edad que incluye BCG (brazo izquierdo) al nacer (Tabla 1).

\section{Pacientes y Método}

Paciente 1: Fecha nacimiento (FN) 6 de diciembre de 1994, sexo femenino. Hija única producto de embarazo y parto de término normal con peso de $3.470 \mathrm{~g}$ y talla de $50 \mathrm{~cm}$.

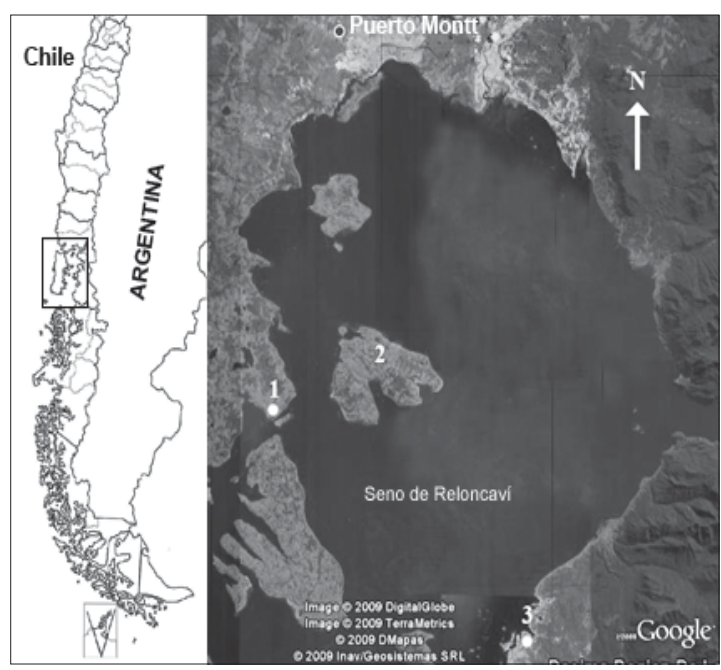

Figura 1. Lugar de residencia en la Región de Los Lagos, Chile de los 5 pacientes de este artículo. Además de Puerto Montt, se señalan con un número las siguientes localidades de residencia: 1. San Agustín; 2. Isla Huar y 3. Aulén.

Tabla 1. Caracterización de 5 pacientes con infección diseminada por BCG

\begin{tabular}{|c|c|c|c|c|}
\hline Paciente & Sexo & $\begin{array}{l}\text { Edad a } \\
\text { la consulta }\end{array}$ & Infección (ubicación y microorganismo) & $\begin{array}{l}\text { Residencia } \\
\text { en X Región, } \\
\text { Chile }\end{array}$ \\
\hline 1 & $\mathrm{~F}$ & 11 meses & $\begin{array}{l}\text { Adenitis axilar, supraclavicular y cervical izquierda BCG. } \\
\text { Candidiasis orofaríngea, ungueal, esofágica. } \\
\text { Meningitis bacteriana Gram (-), no especificada. } \\
\text { Diseminación retroperitoneal y abdominal BCG. }\end{array}$ & Puerto Montt \\
\hline 2 & M & $\begin{array}{l}6 \text { meses } \\
2 \text { años } 4 \text { meses }\end{array}$ & $\begin{array}{c}\text { Adenitis axilar, supraclavicular y cervical izquierda BCG. } \\
\text { Candidiasis orofaríngea. } \\
\text { Adenitis axilar derecha e izquierda BCG. } \\
\text { Testicular, renal, fístula rectovesical ¿BCG? } \\
\text { Adenitis ilíaca, inguinal, peritoneal BCG. } \\
\text { Absceso psoas fistulizado a zona sacra BCG. }\end{array}$ & Puerto Montt \\
\hline 3 & M & 7 meses & $\begin{array}{l}\text { Adenitis axilar izquierda BCG. } \\
\text { Adenitis cervical izquierda y pectoral BCG. } \\
\text { Candidiasis orofaríngea. } \\
\text { Diseminación retroperitoneal y multiorgánica BCG }\end{array}$ & $\begin{array}{l}\text { San Agustín } \\
\text { Calbuco }\end{array}$ \\
\hline 4 & $\mathrm{~F}$ & 3 meses & $\begin{array}{l}\text { Adenitis axilar izquierda BCG. } \\
\text { Adenitis zona pectoral BCG. } \\
\text { Candidiasis orofaríngea. }\end{array}$ & Aulén, Palena \\
\hline 5 & $\mathrm{~F}$ & 4 meses & $\begin{array}{l}\text { Adenitis axilar, supraclavicular y cervical izquierda BCG. } \\
\text { Linfonodos mesentéricos. }\end{array}$ & Isla Huar \\
\hline
\end{tabular}


Consultó a los 11 meses de edad por adenopatía axilar izquierda de $4 \times 4 \mathrm{~cm}$, fistulizada drenando caseum, con baciloscopía y cultivos positivos a bacilos ácido alcohol resistentes (BAAR) (+++) que corresponde a Mycobacterium bovis-BCG resistente a Rifampicina (R) y Cicloserina (Cs).

Se trató inicialmente con aseo quirúrgico seriado, seguido de terapia antituberculosa (TBC), esquema primario simplificado chileno $1996^{6}$, que se prolongó por 8 meses (Tabla 2). Al $5^{\circ}$ mes de tratamiento, aparecieron nuevas adenomegalias en regiones axilar, cervical y supraclavicular izquierdas (Figuras 2 y 3). El examen directo informó BAAR y cultivo positivos a incontables colonias BCG, además de candidiasis orofaríngea y ungueal. A la edad de 1 año y 11 meses se derivó a centro de referencia Santiago Chile, Hospital San Borja-Arriarán (HSBA) para completar su estudio. Los exámenes inmunológicos fueon normales (Tablas 3 y 4), inclu- yendo estudio fagocitario (Nitroblue tetrazolium [NBT] basal $18 \%$ e inducido $57 \%$, adherencia de polimorfonucleares [PMN] $68 \%$, índice fagocitario 2,19 y porcentaje de muerte $51 \%$ ). Panendoscopía digestiva mostró candidiasis esofágica extensa y la ecotomografía abdominal fue normal.

En HSBA se cambió el tratamiento (Tabla 2) agregando Nistatina oral, con lo que disminuyeron los signos clínicos. A los dos meses de tratamiento presentó meningitis bacteriana, con hemocultivo positivo a bacteria Gram (-), no lográndose precisar el germen, además de reagudización de la candidiasis oral, esofágica y ungueal, y adenomegalia axilar izquierda. Se trató con Ceftriaxona endovenoso (ev) por 10 días, más Sulbactam-ampicilina ev por 14 días, gamaglobulina ev 2 g/kg/día por 2 días, Anfotericina B ev durante 21 días y Ketoconazol oral por 6 meses. Se mantuvo el tratamiento con Isoniacida

Tabla 2. Edad a la consulta, sexo, sensibilidad BCG, tratamientos, duración y evolución en 5 pacientes con infección diseminada por BCG

\begin{tabular}{|c|c|c|c|c|c|}
\hline $\begin{array}{c}\text { Edad a } \\
\text { la consulta }\end{array}$ & Sexo & $\begin{array}{l}\text { Sensibilidad } \\
\text { BCG }\end{array}$ & $\begin{array}{c}\text { Tratamiento } \\
\text { infección BCG }\end{array}$ & Duración & $\begin{array}{c}\text { Evolución y } \\
\text { edad }\end{array}$ \\
\hline 11 meses & $\mathrm{F}$ & $\begin{array}{c}\text { Resistente a } \\
\text { R y Cs }\end{array}$ & $\begin{array}{c}\text { H, R, Z }(5,10,25) \mathrm{mg} / \mathrm{kg} / \text { día } \\
\text { H, R }(15,10) \mathrm{mg} / \mathrm{kg} \text { bisemanal } \\
\text { H, E, E t, S }(5,20,15,15) \mathrm{mg} / \mathrm{kg} / \text { día } \\
\text { H, Z, E, E t, Clofazimine } \\
\text { H, E, E t }\end{array}$ & $\begin{array}{l}30 \text { días } \\
10 \text { meses } \\
2 \text { meses } \\
3 \text { meses } \\
\text { Hasta } \\
\text { fallecimiento }\end{array}$ & $\begin{array}{c}\text { Fallece a los } \\
3 \text { años } 9 \text { meses }\end{array}$ \\
\hline $\begin{array}{c}6 \text { meses } \\
2 \text { años } 4 \text { meses }\end{array}$ & M & $\begin{array}{l}\text { Resistente a } \\
\text { Cs y Z } \\
\text { (ganglio) } \\
\text { Resistente a } \\
\text { Cs, Z y R } \\
\text { (orina) }\end{array}$ & $\begin{array}{c}\text { H, R, Z }(5,10,25) \mathrm{mg} / \mathrm{kg} / \mathrm{día} \\
\text { H, R }(15,10) \mathrm{mg} / \mathrm{kg} \text { bisemanal } \\
8 \text { meses sin terapia } \\
\text { H, R, E, S }(5,10,20,15) \mathrm{mg} / \mathrm{kg} / \mathrm{día} \\
\text { H, E, E t, S }(5,10,15,15) \mathrm{mg} / \mathrm{kg} \\
\text { bisemanal } \\
\text { R, E, Et, Clofazimine }\end{array}$ & $\begin{array}{c}30 \text { días } \\
5 \text { meses } \\
2 \text { meses } \\
5 \text { meses } \\
\text { Hasta } \\
\text { fallecimiento }\end{array}$ & $\begin{array}{l}\text { Fallece a los } \\
7 \text { años }\end{array}$ \\
\hline 7 meses & M & $\begin{array}{l}\text { Resistente a Cs } \\
\quad \text { (ganglio) }\end{array}$ & $\begin{array}{c}\text { H (5) mg/kg/día } \\
\text { H, R, Z }(5,10,25) \mathrm{mg} / \mathrm{kg} / \text { día } \\
\text { H, R }(15,10) \mathrm{mg} / \mathrm{kg} \text { bisemanal }\end{array}$ & $\begin{array}{l}2 \text { meses } \\
2 \text { meses } \\
5 \text { meses } \\
\text { (probable que no } \\
\text { asiste a control) }\end{array}$ & $\begin{array}{c}\text { Fallece a } \\
1 \text { año } 11 \text { meses }\end{array}$ \\
\hline 3 meses & $\mathrm{F}$ & $\begin{array}{l}\text { No se logró } \\
\text { tipificar }\end{array}$ & $\begin{array}{c}\text { H, R, Z }(5,10,25) \mathrm{mg} / \mathrm{kg} / \text { día } \\
\mathrm{H}, \mathrm{R}(15,10) \mathrm{mg} / \mathrm{kg} \text { bisemanal } \\
5 \text { meses sin terapia } \\
\text { H, R, Z }(5,10,25) \mathrm{mg} / \mathrm{kg} / \text { día } \\
\text { H, R }(15,10) \mathrm{mg} / \mathrm{kg} \text { bisemanal }\end{array}$ & $\begin{array}{l}2 \text { meses } \\
4 \text { meses } \\
2 \text { meses } \\
4 \text { meses }\end{array}$ & $\begin{array}{l}\text { Asintomática } \\
\text { a los } 3 \text { años } \\
3 \text { meses } \\
\text { Último control } \\
\text { Marzo } 2006\end{array}$ \\
\hline 4 meses & $\mathrm{F}$ & Sensible & $\begin{array}{c}\text { H, R }(5,10) \mathrm{mg} / \mathrm{kg} / \text { día } \\
\text { H, R, Z, E }(15,20,20,20) \mathrm{mg} / \mathrm{kg} / \text { día } \\
24 \text { días sin terapia } \\
\text { H, R, Z, E }(15,20,20,20) \mathrm{mg} / \mathrm{kg} / \text { día } \\
\text { H, R, E }(15,20,20) \mathrm{mg} / \mathrm{kg} / \text { día }\end{array}$ & $\begin{array}{l}30 \text { días } \\
30 \text { días } \\
4 \text { meses } \\
5 \text { meses }\end{array}$ & $\begin{array}{c}\text { Asintomática a } \\
1 \text { año } 7 \text { meses } \\
\text { Último control } \\
\text { Marzo } 2008\end{array}$ \\
\hline
\end{tabular}

R: rifampicina; Cs: cicloserina; Z: pirazinamida; H: isoniacida; Et: etionamida; E: etambutol; S: estreptomicina. 


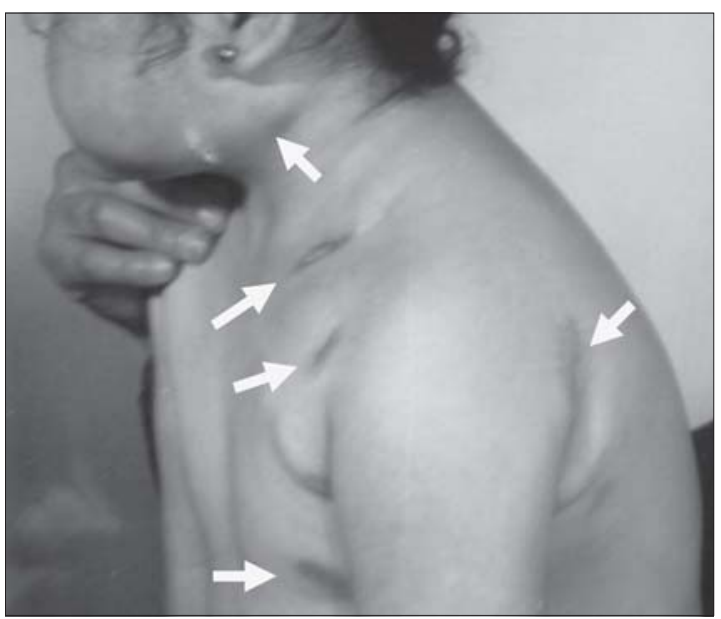

Figura 2a. La fotografía corresponde al perfil de la paciente $n^{\circ} 1$. Se observa: adenopatía preauricular, adenopatías axilares anterior y posterior y la fistulización de un ganglio en posición supraclavicular izquierda (flechas)

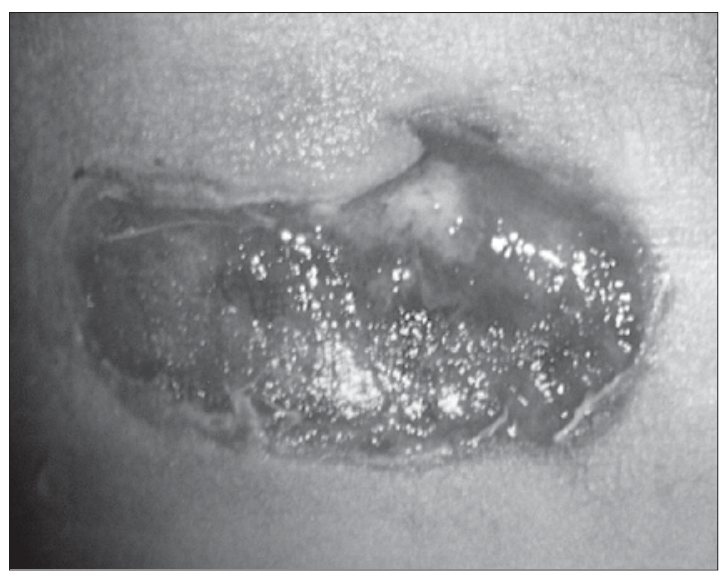

Figura 3a. La fotografía corresponde al acercamiento de lesión de región dorsolumbar del paciente $n^{\circ} 2$. Se observa tejido de granulación y caseum.

(H), Etambutol (E), Etionamida (Et) por 7 meses, más Clofazimine $50 \mathrm{mg}, 2$ veces por semana durante 3 meses. Diez días después de finalizar el tratamiento, reaparecieron las adenopatías axilares, cervicales, supraclaviculares y dorsales con reagudización de la candidiasis oral. Se trató sólo con drenajes y sin antimicrobianos por 5 meses. Reinició terapia los últimos diez meses de vida. La cintigrafía ósea a la edad de 3 años y 6 meses reveló compromiso de costilla y la biopsia ganglionar detectó una linfadenitis BCG con BAAR y cultivos persistentemente positivos. El estudio histopatológico mostró poca di-

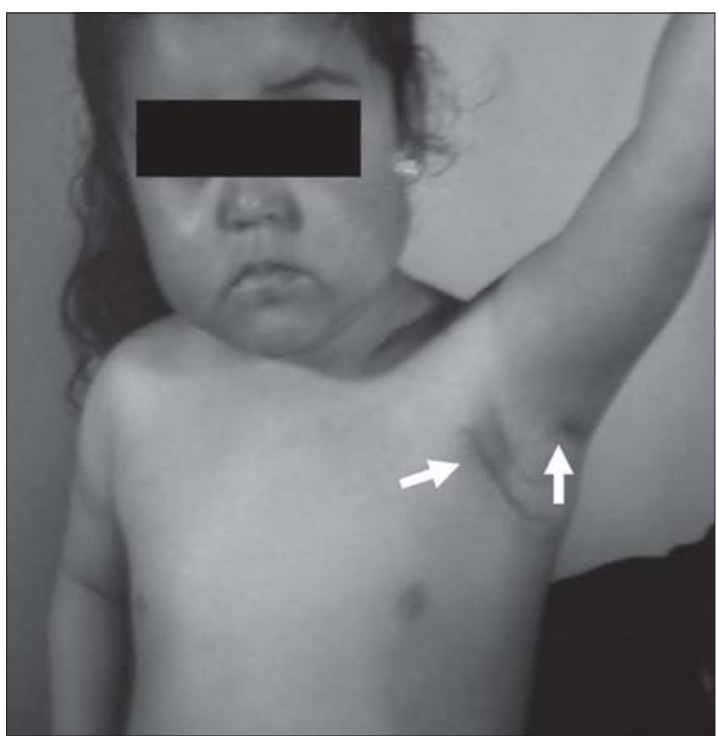

Figura 2b. Toma de frente de la misma paciente $n^{\circ} 1$ permitiendo la visualización de la región axilar izquierda.

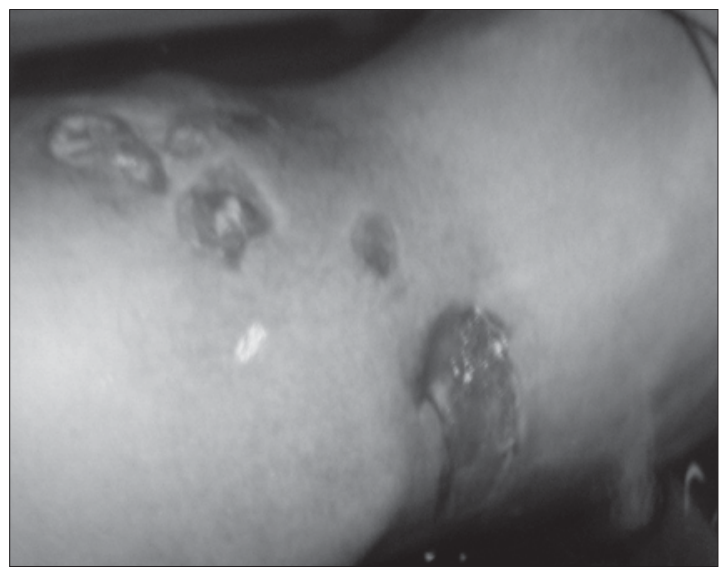

Figura 3b. La fotografía corresponde a la región dorsolumbar del paciente $n^{\circ} 2$. Se observan múltiples lesiones granulomatosas con material caseoso, progresivas y sin signos de cicatrización.

ferenciación con aspecto "lepromatoso" siendo informado como: "tejido aparentemente linfoide de arquitectura extensamente alterada, con folículos inaparentes y depleción marcada de linfocitos; en su reemplazo se observan vastas zonas de histiocitos grandes y pálidos, en parte fusados de citoplasma granular con inclusiones basófilas, sin pleomorfismo o mitosis. No se observan células gigantes multinucleadas, granulomas, necrosis de caseificación, ni focos de calcificación". En sus últimos tres meses de vida presentó anorexia, anemia severa, compromiso del estado general, diseminación a ganglios retro- 
Tabla 3. Exámenes inmunológicos básicos en 5 pacientes con infección diseminada por BCG

\begin{tabular}{|c|c|c|c|c|c|c|c|c|c|c|c|}
\hline Caso & $\begin{array}{l}\text { PPD L } \\
2 \text { UT }\end{array}$ & Leucocitos* & $\begin{array}{c}* \% \\
\text { neutró- } \\
\text { filos } \\
(50-60)\end{array}$ & $\begin{array}{c}\% \\
\text { linfo- } \\
\text { citos } \\
(30-50)\end{array}$ & $\begin{array}{c}\mathrm{IgG} \\
\mathrm{mg} / \mathrm{dL} \\
(800- \\
2.040)\end{array}$ & $\begin{array}{c}\text { IgM } \\
\text { mg/dL } \\
(50-320)\end{array}$ & $\begin{array}{c}\text { IgA } \\
\text { mg/dL } \\
(85-450)\end{array}$ & $\begin{array}{c}\text { IgE } \\
\text { mg/dL } \\
(<100)\end{array}$ & $\begin{array}{c}\mathrm{C} 3 \\
\mathrm{mg} / \mathrm{dL} \\
(86-206)\end{array}$ & $\begin{array}{c}\mathrm{C} 4 \\
\mathrm{mg} / \mathrm{dL} \\
(8-55)\end{array}$ & $\begin{array}{c}\text { VIH } \\
\text { serología } \\
\text { ELISA }\end{array}$ \\
\hline 1 & Negativo & 7.900 & 70 & 30 & 1.891 & 206 & 279 & 92 & 171 & 25 & Negativo \\
\hline 2 & Negativo & 13.500 & 63 & 31 & $\begin{array}{r}922 \\
1.150\end{array}$ & $\begin{array}{l}125 \\
197\end{array}$ & $\begin{array}{l}44,1 \\
126\end{array}$ & $\begin{array}{l}\text { No se } \\
\text { realizó }\end{array}$ & $\begin{array}{l}\text { No se } \\
\text { realizó }\end{array}$ & $\begin{array}{l}\text { No se } \\
\text { realizó }\end{array}$ & Negativo \\
\hline 3 & Negativo & $\begin{array}{l}11.200 \\
33.800 \\
10.800\end{array}$ & $\begin{array}{l}20,7 \\
60,9 \\
46,0\end{array}$ & $\begin{array}{l}79,3 \\
38,1 \\
45,0\end{array}$ & $\begin{array}{l}2.049 \\
1.214\end{array}$ & $\begin{array}{l}272 \\
108\end{array}$ & $\begin{array}{r}30,2 \\
197,8\end{array}$ & $<19,4$ & $\begin{array}{l}\text { No se } \\
\text { realizó }\end{array}$ & $\begin{array}{l}\text { No se } \\
\text { realizó }\end{array}$ & Negativo \\
\hline 4 & Negativo & 13.330 & 38,0 & 52,0 & 1.390 & 252 & $<23,4$ & $\begin{array}{l}\text { No se } \\
\text { realizó }\end{array}$ & $\begin{array}{l}\text { No se } \\
\text { realizó }\end{array}$ & $\begin{array}{l}\text { No se } \\
\text { realizó }\end{array}$ & Negativo \\
\hline 5 & Negativo & 10.900 & 33,0 & 60,0 & 996,4 & 109,3 & 33,3 & $\begin{array}{l}\text { No se } \\
\text { realizó }\end{array}$ & 125,7 & 23,5 & Negativo \\
\hline
\end{tabular}

* Leucocitos: valores normales: 4.000-15.000 (<1 año), 4.000-10.000 (> 1 año).

Tabla 4. Linfocitos y subpoblaciones linfocitarias en 5 pacientes con infección diseminada por BCG

\begin{tabular}{|c|c|c|c|c|c|c|c|c|c|c|c|c|c|}
\hline \multirow[t]{2}{*}{ Caso } & \multicolumn{2}{|c|}{ Recuento } & \multicolumn{2}{|c|}{$\begin{array}{c}\text { Linf. T } \\
\text { CD3 }\end{array}$} & \multicolumn{2}{|c|}{$\begin{array}{c}\text { Linf. T } \\
\text { CD4 }\end{array}$} & \multicolumn{2}{|c|}{$\begin{array}{l}\text { Linf. T } \\
\text { TCD8 }\end{array}$} & \multicolumn{2}{|c|}{$\begin{array}{c}\text { Linf. B } \\
\text { CD19 }\end{array}$} & \multicolumn{2}{|c|}{$\begin{array}{l}\text { Linf. NK } \\
\text { CD16+50 }\end{array}$} & \multirow[t]{2}{*}{$\begin{array}{l}\text { CD4/ } \\
\text { CD8 }\end{array}$} \\
\hline & citos & $\begin{array}{c}\text { citos } \\
\%\end{array}$ & $\%$ & $\mathbf{m m}^{3}$ & $\%$ & $\mathrm{~mm}^{3}$ & $\%$ & $\mathbf{m m}^{3}$ & $\%$ & $\mathrm{~mm}^{3}$ & $\%$ & $\mathbf{m m}^{3}$ & \\
\hline 1 & $\begin{array}{r}20.500 \\
7.900\end{array}$ & $\begin{array}{l}25 \\
30\end{array}$ & $\begin{array}{l}37,7 \\
71\end{array}$ & $\begin{array}{l}1.961 \\
1.682\end{array}$ & $\begin{array}{l}26 \\
54\end{array}$ & $\begin{array}{r}509 \\
1.279\end{array}$ & $\begin{array}{l}8,4 \\
23\end{array}$ & $\begin{array}{l}165 \\
545\end{array}$ & $\begin{array}{l}45 \\
12\end{array}$ & $\begin{array}{r}9.284 \\
285\end{array}$ & $\begin{array}{l}\text { No se } \\
\text { realizó }\end{array}$ & $\begin{array}{l}\text { No se } \\
\text { realizó }\end{array}$ & 0,9 \\
\hline 2 & 12.300 & 36 & 71 & 3.144 & 40 & 1.771 & 26 & 1.151 & 12 & 531 & $\begin{array}{l}\text { No se } \\
\text { realizó }\end{array}$ & $\begin{array}{l}\text { No se } \\
\text { realizó }\end{array}$ & 1,5 \\
\hline 3 & 14.900 & 38 & 56,9 & 3.222 & 33,1 & 1.874 & 23,2 & 1.314 & 25,4 & 1.438 & 15,8 & 895 & 1,4 \\
\hline 4 & 9.100 & 54 & 76,9 & 3.779 & 43,2 & 2.123 & 36,5 & 1.794 & 15,4 & 757 & 9,2 & 452 & 1,2 \\
\hline 5 & 10.000 & 52,3 & 59,8 & 3.127 & 34,0 & 1.778 & 20,8 & 1.087 & 19,1 & 999 & 18,2 & 952 & 1,6 \\
\hline
\end{tabular}

Linf.: Linfocitos. Valores normales: CD3: (54-76\%), (1.600-6.700). CD4: (31-54\%), (1.000-4.600). TCD8: (12-28\%), (400-2.100). CD19: (15-39\%), (600-2.700). CD16+50: (3-17\%), (200-1.200). CD4/CD8: (0,9-2,0).

peritoneales y abdominales, ascitis y edema de hemicuerpo inferior y de brazo izquierdo. Falleció en su domicilio a los 3 años y 9 meses de edad. No se efectuó autopsia.

Paciente 2: FN 11 de febrero de 1992. Sexo masculino. Hijo único de padres jóvenes. Producto de embarazo y parto de término normal con peso de $3.000 \mathrm{~g}$ y talla de $50 \mathrm{~cm}$. Consultó a los 6 meses de edad por adenitis axilar y supraclavicular izquierda y derecha fistulizadas. La baciloscopía muestra BAAR positivo y cultivo $M$. bovis-BCG. La biopsia reveló "necrosis focal, moderada fibrosis y desarrollo de granulomas epitelioídeos con células gigantes de
Langerhans". Luego de múltiples drenajes quirúrgicos se derivó a los 2 años y 4 meses a Centro de Referencia en Santiago de Chile: HSBA. Se trató con antimicrobianos según esquema primario simplificado (Tabla 2), con resolución completa de las adenopatías.

Los exámenes inmunológicos resultaron normales (Tablas 3 y 4), incluyendo estudio fagocitario (Migration inhibitor factor [factor inhibidor de la migración: MIF], Antígeno PPD 33\%, MIF PHA [fitohemoglutinina] 66\%, NBT espontáneo 0,312 y reducción con zimozan 0,372 , índice fagocítico 2,4 , porcentaje de muerte $40,8 \%$, quimiotaxis al azar $0,65 \mathrm{y}$ dirigida 1,16 , factor quimiotáctico 1,54$)$. 
Ocho meses después de suspender tratamiento presentó aumento de volumen testicular derecho abscedado, cuya biopsia reveló tejido de granulación inespecífico. La tomografía axial computada (TAC) abdominal demostró hidronefrosis izquierda, múltiples adenopatías ilíacas, inguinales, peritoneales y pélvicas. Un mes después apareció fístula rectovesical que se confirmó con rectoscopía, ultrasonografía y enema baritado. La biopsia de mucosa rectal detectó tejido inflamatorio inespecífico y el examen de orina reveló BAAR (+++). El paciente reinició tratamiento con 4 drogas anti TBC; Estreptomicina (S), Rifampicina (R), Etambutol (E) y Pirazinamida $(Z)$ por 3 meses; posteriormente con R, E y Z y refuerzos ocasionales de S. Se agregó Clofazimine $200 \mathrm{mg} 1 \mathrm{vez}$ al mes y 50 mg en días alternos hasta Febrero de 1999 (Tabla 2).

Pese al tratamiento permanente presentó reactivación de la fístula rectovesical y progresión del daño renal, con examen directo y cultivo de orina persistentemente positivos a BAAR. En Marzo 1998 se extirpó el riñón izquierdo y en el procedimiento se evidenció absceso frío en región distal del psoas derecho, que se había fistulizado con material caseoso y tejido de granulación exhuberante, que abarcaba toda la zona lumbosacra y perianal, con cultivos positivos a BAAR (Figuras 3 a y b).

Se trató con Interferón gama $50 \mathrm{mg} / \mathrm{m}^{2}$ subcutáneo por 3 semanas, con mala tolerancia, presentando hipertermia de $40{ }^{\circ} \mathrm{C}$, calofríos y náuseas, sin modificación de la evolución clínica. En diciembre 1998 se realizó colostomía de descarga y se injertó zona lumbosacra, pero presentó rechazo del injerto. En los últimos 3 meses presentó anorexia marcada, anemia severa, caquexia extrema y estatus convulsivo. Falleció a la edad de 7 años con cuidados paliativos en su domicilio. No se efectuó autopsia.

Paciente 3: FN 17 de febrero de 2002, sexo masculino. Consultó a los 7 meses de edad por aumento de volumen axilar izquierdo de 4 a 5 $\mathrm{cm}$, de un mes de evolución, efectuándose drenaje. La baciloscopía de la lesión dio como resultado BAAR positiva con cultivos positivos a M. bovis-BCG resistente a Cs. Se trató con monoterapia $(\mathrm{H})$ durante 2 meses (Tabla 2).

Al segundo mes de tratamiento se encontró adenomegalia axilar izquierda mayor de $10 \mathrm{~cm}$ de diámetro.

Los estudios inmunológicos se enumeran en las Tablas 3 y 4 . No se realizó estudio fagocitario.

Se cambió a tratamiento según esquema pri- mario simplificado (Tabla 2), que es bien tolerado, con lo que desaparecieron las adenopatías, persistiendo sólo la cicatriz de fistulización. Siete días después del inicio de la terapia bisemanal reapareció el aumento de volumen axilar izquierdo, pese a lo cual continuó con igual esquema de tratamiento hasta completar 40 dosis.

Cuatro meses después de finalizar tratamiento consultó por lesiones supuradas del tronco de dos meses de evolución, con destrucción de la piel en forma de sacabocado, con áreas de necrosis y tejido granulomatoso en tronco y axila izquierda.

Se hospitalizó grave, febril $\left(39^{\circ} \mathrm{C}\right)$, enflaquecido (peso corporal en percentil 7) con poliadenopatías cervicales, lesiones quísticas fluctuantes en axila izquierda y hemitórax izquierdo, hepatomegalia de tres $\mathrm{cm}$, y candidiasis oral. En las lesiones de la piel se identificaron BAAR. Un hemocultivo fue positivo a neumococo sensible a los antibióticos usados (Ceftriaxona y Clindamicina ev).

Falleció a los 2 días de hospitalización. La autopsia demostró diseminación BCG generalizada, con siembra de BAAR peritoneal y multiorgánica. El informe histopatológico se describió así: "ganglios axilares con acentuada necrosis, arquitectura distorsionada. No se reconocen granulomas".

Paciente 4: FN 28 de octubre 2002, sexo femenino. Consultó a los 3 meses de edad por adenopatía axilar izquierda de 15 días de evolución, piel violácea y fistulización espontánea, drenando material caseoso. Se efectuó aseo quirúrgico y se envió a cultivo que resultó negativo para bacterias y micobacterias. Un mes después presentó brusco crecimiento de adenopatía axilar izquierda, de $10 \mathrm{~cm}$ de diámetro que compromete la zona pectoral hasta pliegue axilar posterior izquierdo, presenta un área fistulizada de 1 $\mathrm{cm}$ de diámetro, con tejido de granulación y material caseoso, además de extensa candidiasis oral. El examen directo de la lesión fue altamente positivo (+++) para BAAR aunque no fue posible tipificar.

Los exámenes inmunológicos resultaron normales (Tablas 3 y 4), incluyendo el estudio fagocitario. Se trató con esquema primario simplificado modificado ${ }^{7}$ obteniéndose regresión casi total de la adenopatía, sólo quedó una pequeña área residual de $1-1,5 \mathrm{~cm}$ de aspecto granulomatoso.

A los 5 meses de finalizar la terapia presentó recidiva con lesión fistulizada con BAAR (+). Se efectuó limpieza quirúrgica y biopsia, reini- 
ciando tratamiento anti TBC durante 6 meses con nueva resolución clínica. En el último control a los 3 años y 3 meses, presentaba sólo una pequeña calcificación de $2 \mathrm{~mm}$ en axila izquierda.

Paciente 5: FN 25 de octubre de 2006, sexo femenino (prima hermana de la paciente 4). El padre y la hermana de 11 años son sanos. La madre presentaba el antecedente de extracción de nódulos en la zona retroauricular derecha y en el dorso de etiología no precisada. La paciente consultó a los 4 meses de vida por adenopatías cervical y axilar izquierda de 1 a $1,5 \mathrm{~cm}$ sin fistulización. Las ecografías cervical y axilar confirmaron la presencia de 4 nódulos supraclaviculares izquierdos y axilares izquierdos con aspecto BCG. La ecografía abdominal mostró la presencia de adenomegalias mesentéricas de hasta $8,3 \mathrm{~mm}$ de diámetro. Se efectuaron tres curetajes, el último por recidiva de aproximadamente $4 \mathrm{~cm}$ de diámetro. El examen directo informó BAAR (-) y el cultivo fue positivo a $M$. bovisBCG.

Los exámenes inmunológicos mostraron resultados normales (Tablas 3 y 4). La radiografía de tórax frontal y lateral era normal. Los estudios del eje interleuquina 12/interferón gama (IL$12 / \mathrm{IFN}-\gamma)$ realizados en sangre luego de la activación por BCG, BCG + IL-12 y BCG + IFN- $\gamma$ detectaron secreción normal de IFN- $\gamma$ y de IL12 (Figura 4).
Inició terapia con dos drogas $(\mathrm{H}, \mathrm{R})$ durante un mes, ampliándose el esquema a 4 drogas $(\mathrm{H}$, $\mathrm{R}, \mathrm{E}, \mathrm{Z})$ por cuatro meses, los que cumplió en forma discontinua por dificultades en el control y de comprensión materna de la enfermedad. Al $5^{\circ}$ mes de tratamiento se suspendió la Pirazinamida, continuando con 3 drogas durante 5 meses en igual dosis y luego con terapia bisemanal hasta completar un año (Tabla 2). La evolución clínica fue satisfactoria hasta el último control (marzo 2008) en que sólo fueron detectables por ecografía adenopatías de 1 a 2 $\mathrm{mm}$ en axila izquierda.

\section{Comentarios}

Se describen los primeros pacientes con infección diseminada por $M$. bovis-BCG en la región de Los Lagos de Chile. Esta región está situada en la región patagónica, y comprende las provincias de Osorno, Llanquihue, Chiloé y Palena. Geográficamente está compuesta de territorio continental y 50 islas, con una superficie aproximada de $48.583,6 \mathrm{~km}^{2}$ y una población de 716.769 habitantes (Figura 1). La población se compone fundamentalmente de pobladores chilenos. Aunque no hay antecedentes de consanguinidad en estos pacientes, esta situación geográfica hace sospechar cierto parentesco entre los pobladores de la región.

Nuestros casos clínicos corresponden a efec-

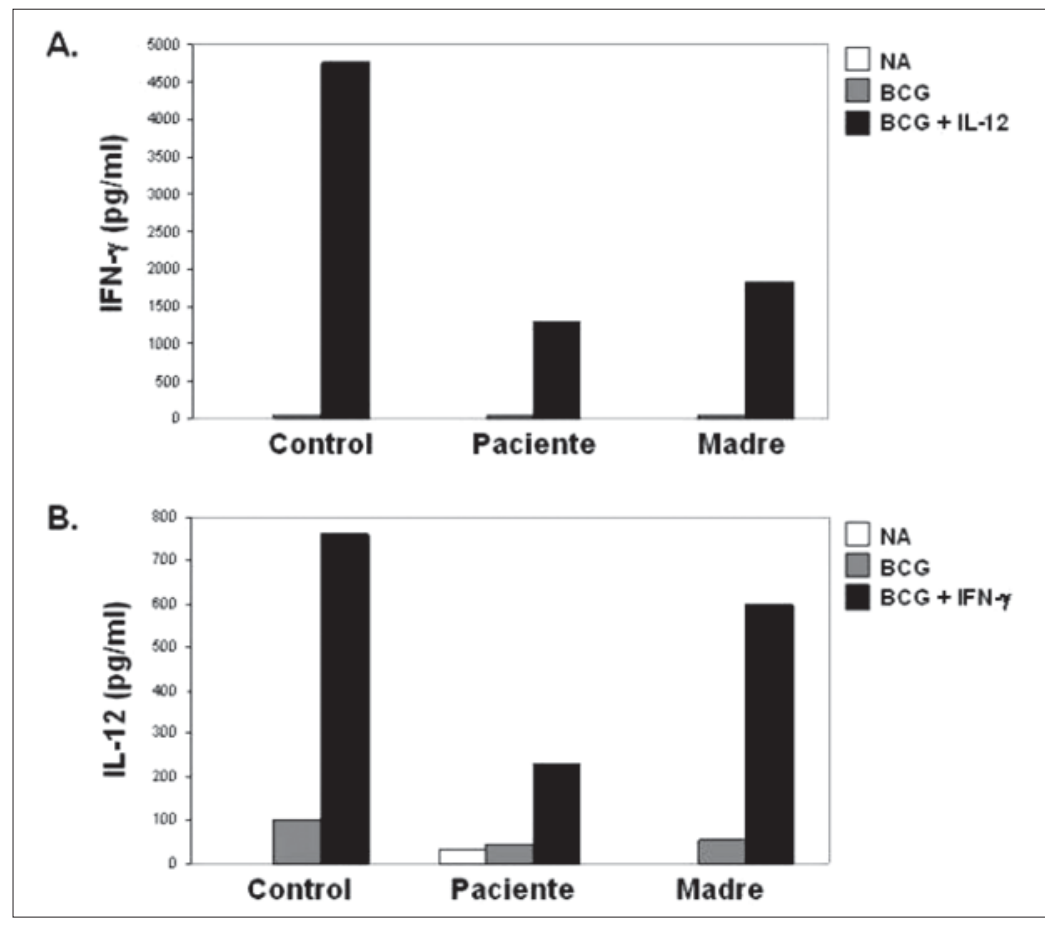

Rev Chil Enf Respir 2009; 25: 29-38
Figura 4. Investigación del eje IL$12 /$ IFN- $\gamma$ en sangre, técnica de ELISA. A. Producción de IFN- $\gamma$ (pg/ml) luego de la activación por BCG, BCG + IL-12 en un control sano, la paciente y la madre. NA: Sin activación. B. Producción de IL-12 (pg/ml) luego de la activación por $\mathrm{BCG}, \mathrm{BCG}+\mathrm{IFN}-\gamma$ en un control sano, la paciente y la madre. NA: Sin activación. Los sobrenadantes fueron recolectados luego de 48 h de la activación y usados para realizar la cuantificación de citoquinas por técnica de ELISA. 
tos adversos severos de la vacuna BCG neonatal, en pacientes aparentemente sanos, en los cuales no se ha identificado el defecto inmune subyacente, con evoluciones clínicas diferentes lo que probablemente corresponde a una suma de factores tales como distintas alteraciones de la respuesta inmune, tratamientos inadecuados e ineficaces o abandono de tratamiento, secundario a falta de comprensión de la familia, o negativa a continuar terapia por razones culturales y sociales.

La vacuna BCG neonatal es usada en países en desarrollo con alta prevalencia de enfermedad TBC, por su eficacia documentada en prevenir las formas letales en lactantes y niños sin enfermedad por virus de inmunodeficiencia humana (VIH), aunque no previene la infección de otras localizaciones. La OMS la ha recomendado independientemente de la exposición a VIH, excepto si el niño tiene VIH sintomático.

El Mycobacterium bovis variedad BCG (Bacilo de Calmette-Guérin) es un microorganismo de baja patogenicidad que induce respuesta inmune tipo Th1 que confiere protección cruzada contra el bacilo de $\mathrm{Koch}^{8}$.

Se han descrito los siguientes efectos adversos de la vacunación BCG:

- Complicaciones en el sitio de inyección.

- Adenitis fistulizada local.

- Enfermedad diseminada regional o sistémica.

Las dos primeras se han relacionado a la cepa BCG utilizada, dosis, vía y técnica de la administración de la vacuna, todos ellos factores que determinan la carga bacilar ${ }^{9-11}$.

La infección diseminada por $M$. bovis-BCG sugiere un compromiso de la inmunidad del huésped $^{12-14}$. Se ha descrito en pacientes inmunodeficientes secundarios (VIH, cáncer) ${ }^{12-18}$ y primarios (déficit cuantitativo o cualitativo de linfocitos $\mathrm{T}$ como inmunodeficiencia común variable, síndrome de hiper IgE, síndrome de Di George, síndrome de hiper IgM y enfermedad granulomatosa crónica) ${ }^{12-14,19-22}$ y en el síndrome de susceptibilidad mendeliana a las infecciones micobacterianas-SPMIM ${ }^{1-5,23}$. Este síndrome se define por enfermedad clínica severa, ya sea diseminada o localizada y recurrente causada por especies micobacterianas débilmente virulentas como micobacterias del medio ambiente, M. bovis-BCG y en algunos casos a M. tuberculosis; en la mitad de los casos se halla una asociación con infecciones por Salmonella, de localización extraintestinal; no hay otra infección asociada ${ }^{1-5}$.

El resultado de los exámenes inmunológicos en los pacientes con este síndrome generalmente es normal. En el 50\% de los pacientes descritos en la literatura pueden identificarse alteraciones genéticas y moleculares del eje IFN- $\gamma / \mathrm{IL} 12 /$ 23 , de las cuales se han descrito 6 genes y 13 alteraciones $^{3-5}$, las que se expresan clínicamente en forma diferente, siendo las de peor pronóstico los defectos completos de las cadenas 1 ó 2 del receptor de IFN- $\gamma$.

El estudio inmunológico de los pacientes requiere exámenes especializados que no están fácilmente disponibles en el sistema público de salud, aunque existe la posibilidad de efectuarlos en centros nacionales e internacionales en el contexto del estudio de Inmunodeficiencias primarias (LAGID). Actualmente, existe además la posibilidad de enviar muestras para el estudio del circuito IL12/23- IFN- $\gamma$ a Francia.

La evolución y el pronóstico de las formas diseminadas se han relacionado con la alteración histopatológica en las muestras de biopsia o autopsia, las que a su vez dependen del defecto inmunológico de base y de la etapa de la enfermedad $^{19,24}$. La respuesta al tratamiento depende también del defecto de base, de la precocidad del inicio del tratamiento, de su duración y de las drogas usadas ${ }^{25-28}$.

Al no existir un esquema de tratamiento validado, los esquemas terapéuticos usados se basaron en las pautas de tratamiento de adenitis TBC, incluyendo pirazinamida, los que se modificaron luego de conocer informes del tipo de micobacterio y de la sensibilidad, lo que puede haber influido en la evolución clínica de los pacientes, junto al defecto inmunológico probable de base.

Aunque el estudio inmunológico realizado es normal, dadas las características clínicas y las alteraciones histopatológicas de nuestros pacientes, los diagnósticos diferenciales en los pacientes 1 y 3 pueden corresponder a deficiencia completa de una de las cadenas del receptor de interferón gama. En el caso 2 el fenotipo clínico se parece a una enfermedad granulomatosa crónica, entidad clínica que también se asocia a enfermedades micobacterianas. Los casos 4 y 5 relacionados familiarmente (padres son primos hermanos), podrían corresponder a otras deficiencias parciales de componentes del eje IL12/ IFN- $\gamma$ u otros defectos no identificados.

Considerando la diferencia en pronóstico y evolución de las distintas etiologías es importante realizar el estudio completo de todas las formas clínicas con excepción de las adenitis ipsilaterales pequeñas que se resuelven espontáneamente o con medidas de drenaje local y precisar 
la terapia óptima, tanto en medicamentos como en la duración de éste por lo que enfatizamos la necesidad de que todos los pacientes con alguna reacción adversa a la vacuna BCG sean derivados a médicos especializados para poder llegar a un diagnóstico genético y molecular que permita un tratamiento adecuado basado en la fisiopatología.

En conclusión, describimos los aspectos clínicos de 5 pacientes aparentemente sanos que presentan reacción adversa severa a vacuna BCG neonatal, los que cumplen con los criterios del Síndrome de predisposición mendeliana a enfermedades micobacterianas, tres de ellos con evolución letal y dos relacionados familiarmente con buen pronóstico vital hasta la fecha del seguimiento.

\section{Agradecimientos}

Los autores desean agradecer en forma muy especial al Dr. Jean Laurent Casanova por el estímulo al estudio genético y publicación de estos casos y a Daniel Noriega Azócar por su importante ayuda en la elaboración del manuscrito e imágenes.

\section{Bibliografía}

1.- CASANOVA J L, BLANCHE S, EMILE J F, JOUANGUY E, LAMHAMEDIS S, ALTARE F, et al. Idiopathic disseminated bacillus Calmette-Guérin infection: A French national retrospective study. Pediatrics 1996; 98: 774-8.

2.- JOUANGUY E, LAMHAMEDI-CHERRADI S, LAMMAS D, DORMAN S E, FONDANECHE M C, DUPUIS S, et al. A human IFNGR1 small deletion hotspot associated with dominant susceptibility to mycobacterial infection. Nat Genet 1999; 21: 370-8.

3.- REMUS N, REICHENBACH J, PICARD C, RIETSCHEL C, WOOD P, LAMMAS D, et al. Impaired interferon gamma-mediated immunity and susceptibility to mycobacterial infection in childhood. Pediatr Res 2001; 50: 8-13.

4.- FILIPE-SANTOS O, BUSTAMANTE J, CHAPGIER A, JOGT G, DE BEAVCOUDREY L, FEINBERG J, et al. Inborn errors of IL-12/23- and IFN-gamma-mediated immunity: molecular, cellular, and clinical features. Semin Immunol 2006; 18: 347-61.

5.- PEDRAZA S, ALDERA R, HERRERA MT, TORRES M, BUSTAMANTE J. Infecciones por Mycobacterium y Salmonella en pacientes con inmunodeficiencias asociadas al circuito IL-12/IL-23-/IFN- $\gamma$ : aspectos biológicos y clínicos. Enf Inf Microbiol 2007; 27: 88-9.

6.- PROGRAMA NACIONAL DE CONTROL DE LA TUBERCULOSIS. Ministerio de Salud, República de Chile. Publicación Oficial. 1996.

7.- PROGRAMA NACIONAL DE CONTROL DE LA TUBERCULOSIS. Ministerio de Salud, República de Chile. Publicación Oficial. 2005.
8.- MARCHANT A, GOETGHEBUER T, OTA MO, WOLFE I, CEESAY S J, DE GROOTE D, et al. Newborns develop a Th1-type immune response to Mycobacterium bovis bacillus Calmette-Guérin vaccination. J Immunol 1999; 163: 2249-55.

9.- FINE P E M, CARNEIRO I A M, MILSTEIN J B, CLEMENTS $\mathrm{C}$ J. Issues relating to the use of BCG immunization programmes-a discussion document. World Health Organization (WHO) document no. WHO/V\&B/99.23. Geneva: WHO, 1999.

10.- MILSTEIN J B, GIBSON J J. Quality control of BCG by WHO: a review of factors that may influence vaccine effectiveness and safety. Bull World Health Organ 1990; 68: 93-108.

11.- LOTTE A, WASZ-HOCKERT O, POISSON N, ENGBAEK H, LANDMANN H, QUAST U, et al. Second IUATLD study on complications induced by intradermal BCG-vaccination. Bull Int Union Tuberc Lung Dis 1988; 63: 47-59.

12.- TALBOT E A, PERKINS M D, SILVA S F, FROTHINGHAM R. Disseminated bacille CalmetteGuerin disease after vaccination: case report and review. Clin Infect Dis 1997; 24: 1139-46.

13.- CASANOVA J L, JOUANGUY E, LAMHAMEDI S, BLANCHE S, FISCHER A. Immunological conditions of children with BCG disseminated infection. Lancet 1995; 346 (8974): 581.

14.- BERNATOWSKA E, WOLSKE-KUZNIERZ B, PAC M, KURENKO-DEPTUCH M, ZWOLSKA Z, CASANOVA J L, et al. Disseminated bacillus Calmette-Guérin infection and immunodeficiency. Emerg Infect Dis 2007; 13: 799-801.

15.- O'BRIEN K L, RUFF A J, LOUIS M A, DESORMEAUX J, JOSEPH D J, MCBRIEN M, et al. Bacillus CalmetteGuérin complications in children born to HIV-1infected women with a review of the literature. Pediatrics 1995; 95: 414-8.

16.- HESSELING A C, SCHAAF H S, HANEKOM W A, BEYERS N, COTTON M F, GIE R P, et al. Danish bacille Calmette-Guérin vaccine-induced disease in human immunodeficiency virus-infected children. Clin Infect Dis 2003; 37: 1226-33.

17.- HESSELING A C, MARAIS B J, GIE R P, SCHAAF H S, FINE P E, GODFREY-FAUSSETT P, ET AL. The risk of disseminated Bacille Calmette-Guerin (BCG) disease in HIV-infected children. Vaccine 2007; 25: 14-8.

18.- HESSELING A C, RABIE H, MARAIS B J, MANDERS M, LIPS M, SCHAAF H S, et al. Bacille CalmetteGuérin vaccine-induced disease in HIV-infected and HIVuninfected children. Clin Infect Dis 2006; 42: 54858.

19.- ABRAMOWSKY C, GONZÁLEZ B, SORENSEN R U. Disseminated bacillus Calmette-Guérin infections in patients with primary immunodeficiencies. Am J Clin Pathol 1993; 100: 52-6.

20.- GONZÁLEZ B, MORENO S, BURDACH R, VALENZUELA MT, HENRÍQUEZ A, RAMOS MI, et al. Clinical presentation of Bacillus Calmette-Guérin infections in patients with immunodeficiency syndromes. Pediatr Infect Dis J 1989; 8: 201-6.

21.- PASIC S, LILIC D, PEJNOVIC N, VOJVODIC D, SIMIC R, ABINUM M. Disseminated bacillus CalmetteGuérin infection in a girl with hyperimmunoglobulin E syndrome. Acta Paediatr 1998; 87: 702-4.

22.- PASIC S. Local bacillus Calmette-Guérin infection in hyperimmunoglobulin E syndrome. Acta Paediatr 2002; 91: 1271-2. 
23.- JOUANGUY E, LAMHAMEDI-CHERRADI S, ALTARE F, FONDANECHE M C, TUERLINCKX D, BLANCHE S, et al. Partial interferon -gamma receptor 1 deficiency in a child with tuberculoid bacillus Calmette-Guérin infection and a sibling with clinical tuberculosis. J Clin Invest 1997; 100: 2658-64.

24.- EMILE J F, PATEY N, ALTARE F, LAMHAMEDI S, JOUANGUY E, BOMAN F, et al. Correlation of granuloma structure with clinical outcome defines two types of idiopathic disseminated BCG infection. J Pathol 1997; 181: 25-30.

25.- ROSENZWEIG S D, YANCOSKI J, BERNASCONI A, KRASOVEC S, MARCIANO B E, CASIMIR L, et al. Thirteen years of culture-positive $M$. bovis-BCG infection in an IL-12Rbetal deficient patient: treatment and outcome. J Infect 2006; 52: e69-72.

26.- HESSELING A C, SCHAAF H S, VICTOR T, BEYERS
N, MARAIS V J, COTTON M F, et al. Resistant Mycobacterium bovis bacillus Calmette-Guérin disease: Implications for management of bacillus CalmetteGuérin disease in human immunodeficiency virusinfected children. Pediatr Infect Dis J 2004; 23: 476-9.

27.- SANAL O, MORGAN G, GÖÇMEN A, NOVELLI V, KLEIN N, TEZCAN I, et al. Isolated cutaneous response to granulocyte-monocyte colony stimulating factor in fatal idiopathic disseminated bacillus Calmette-Guérin infection. Eur J Pediatr 2000; 159: 149-52.

28.- ULRICHS T, FIESCHI C, NEVICKA E, HAHN H, BREZINA M, KAUFMANN S H, et al. Variable outcome of experimental interferon-gamma therapy of disseminated bacillus Calmette-Guérin infection in two unrelated interleukin-12 Rb1- deficient Slovakian children. Eur J Pediatr 2005; 164: 166-72.

Correspondencia a:

Alexis Strickler Prouvay

Servicio de Pediatría, Hospital de Puerto Montt.

E-mail: astrickler@123.cl 